\title{
Two Stream Instability as a Source of Coronal Heating
}

\author{
Antony Soosaleon*, Blesson Jose \\ SPAP, Mahatma Gandhi University, Kottayam, India \\ Email: *antonysoosaleon@yahoo.com
}

Received 22 January 2015; accepted 29 April 2015; published 4 May 2015

Copyright (C) 2015 by authors and Scientific Research Publishing Inc.

This work is licensed under the Creative Commons Attribution International License (CC BY). http://creativecommons.org/licenses/by/4.0/

CC) (i) Open Access

\begin{abstract}
Recent observation of oscillating the two stream instability (TSI) in a solar type III radio bursts and spatial damping of Langmuir oscillations has made this instability as an important candidate to understand the coronal heating problem. This instability has been studied by several authors for cold plasma found to be stable for high frequencies (greater than plasma frequency $\omega_{p}$ ). In this paper, we prove that this instability is unstable for warm plasma for higher frequencies (greater than plasma frequency $\omega_{p}$ ) and much suitable to study the solar coronal heating problem. We have derived a general dispersion relation for warm plasma and discussed the various methods analyzing the instability conditions. Also, we derived an expression for the growth rate of TSI and analyzed the growth rate for photospheric and coronal plasmas. A very promising result is that the ion temperature is the source of this instability and shifts the growth rate to high frequency region, while the electron temperature does the reverse. TSI shows a high growth rate for a wide frequency range for photosphere plasma, suggesting that the electron precipitation by magnetic reconnection current, acceleration by flares, may be source of TSI in the photosphere. But for corona, these waves are damped to accelerate the ions and further growing of such instability is prohibited due to the high conductivity in coronal plasma. The TSI is a common instability; the theory can be easily modifiable for multi-ion plasmas and will be a useful tool to analyze all the astrophysical problems and industrial devices, too.
\end{abstract}

\section{Keywords}

Coronal Heating, Two Stream Instability, Langmuir Waves, Ion Temperature, Drift Velocity, Photosphere, Fusion Plasma

\footnotetext{
${ }^{*}$ Corresponding author.
} 


\section{Introduction}

Solar corona is hot to the level of million degree kelvin; several mechanisms have been put forward for the last 60 years which is continued as the problem exist. In this paper, we try to add one more to the list of possible mechanisms as a source of coronal heating, which is the streaming instability. Streaming instabilities arise when there is relative velocity between ions and electrons in a plasma. The simplest type of streaming instability is the two stream instability (TSI). This arises in an electron-proton plasma with electrons in relative motion with ions. This type of streaming instability arises in stellar atmospheres, since the stellar plasmas are predominantly electron-proton type. A specific example for this instability is an electron-precipitation related phenomenon in solar chromosphere [1], and also the presence of oscillating TSI in a solar type III radio bursts [2] is a strong experimental support for our theoretical calculation which shows a greater growth rate of TSI in the photosphere. The very important point presented by them is the spatial damping of Langmuir waves induced by the TSI, which is also our conclusion that these waves are damped out in coronal plasma which heats the ions. The streaming instability can also be ignited when a high energy beam of electrons created during the process of reconnection taking place at the site of a solar flare comes down towards the chromosphere. Since the density of chromosphere plasma is high, the energetic electrons from the flare suffer collisions and transfers their energy to electrons and protons and to the small population of heavier ions too. These secondary electrons and ions are accelerated with different speeds [3] [4]. This creates a plasma in which electrons and protons are at different velocities and always electrons drive fast to ions which create the situation for the TSI. The TSI for hot plasmas also arises at chromospheric foot points heating by energetic streams from magnetic reconnection, which is discussed in [5] and [6]. Similar situation happens at helmet streamers, cometary atmospheres and earth magneto sphere, etc.

The electromagnetic wave propagation and instabilities for the counter streaming astrophysical situations for cold plasma have been discussed by several authors [7]-[12], who find them unimportant, because it is unstable for very low frequency (less than plasma frequency $\omega_{p}$ ), but TSI is a high frequency wave and also the growth rate is negligible for cold plasma. But for warm plasma, we find it is unstable for a very wide range of frequencies and even for the frequencies much greater than $\omega_{p}$ and that the growth rate is very large and sensitively depends on the ion (electron) temperatures which is ideal for solar atmosphere.

An interesting result is that the growth rate shifts to the higher frequency region as the ion temperature increases, while the electron temperature shifts it to the lower frequency region. It is the ion temperature that becomes the source of TSI as the high frequency oscillations, for it is a necessary condition presumed for this instability and hence the damping of this instability will heat the ions. Also, this instability depends on the electron temperature, but stabilises the plasma. It is found that the growth rate region shifts towards the low frequency regions as the electron temperature increases in reverse of the shift due to ion temperature. As the kinetic energy of electrons shifts the growth rate to the low frequency region which is nothing, damping of high frequency oscillation results in the heating of ions. The high frequency oscillations are induced by the electric field due to the electron drift which accelerates the ions at the expense of kinetic energy of electrons, and this is true because the increase in drift velocity decreases the instability. This instability is a common one and hence this theory can be applied to any hot electron-proton plasma and can be better for fusion studies too.

\section{Theory}

For the theoretical study of streaming instability in a hot plasma, we consider an electron-proton plasma, with ions assumed stationary and electrons moving with a velocity $v_{0}$ relative to ions. This is same as we assume the observer moving with a stream of ions. We consider hot plasma $K T \neq 0$. For simple analysis we consider the case of zero ambient magnetic field $\left(B_{0}=0\right)$. It can easily shown that the same results can be applied for electrostatic waves along magnetic field.

The linearised equation of motion for protons and electrons are respectively:

$$
\begin{aligned}
& M n_{0} \frac{\partial \boldsymbol{v}_{i 1}}{\partial t}=e n_{0} \boldsymbol{E}_{1}-\gamma_{i} K T_{i} \nabla n_{i 1} \\
& m n_{0} \frac{\partial \boldsymbol{v}_{e 1}}{\partial t}=-e n_{0} \boldsymbol{E}_{1}-\gamma_{e} K T_{e} \nabla n_{e 1}
\end{aligned}
$$

We consider electrostatic waves of the form, 


$$
E_{1}=E \exp [i(k x-\omega t)] \hat{x}
$$

where $\hat{x}$ is in the direction of $\boldsymbol{v}_{0}$ and $\boldsymbol{k}$.

Under these conditions Equation (1) gives

$$
-i \omega M n_{0} v_{i 1} \hat{x}=e n_{0} E \hat{x}-\gamma_{i} K T_{i} i k n_{i 1} \hat{x}
$$

i.e.,

$$
\boldsymbol{v}_{i 1}=\frac{i e}{M \omega} E \hat{x}+\frac{\gamma_{i} K T_{i}}{M \omega} k\left(\frac{n_{i 1}}{n_{0}}\right) \hat{x}
$$

Similarly, Equation (2) gives

$$
\boldsymbol{v}_{e 1}=\frac{-i e}{m} \frac{E}{\left(\omega-k v_{0}\right)} \hat{x}+\frac{\gamma_{e} K T_{e}}{m\left(\omega-k v_{0}\right)} k\left(\frac{n_{e 1}}{n_{0}}\right) \hat{x}
$$

The same results hold good for parallel electrostatic plasma oscillations (i.e., wave propagation parallel to $B_{0}$.) The ion continuity equation for our case is

$$
\frac{\partial n_{i 1}}{\partial t}+n_{0}\left(\nabla \cdot \boldsymbol{v}_{i 1}\right)=0
$$

Linearising this and noting that $\nabla \cdot v_{0}$ and $\nabla n_{0}$ vanishes, and using the value of $v_{i 1}$, we get for protons

$$
n_{i 1}=\frac{k}{\omega} n_{0} v_{i 1}=\left(\frac{i e n_{0} k}{M \omega^{2}} E+\frac{k^{2} \gamma_{i} K T_{i}}{M \omega^{2}} n_{i 1}\right)
$$

Similarly the electron continuity equation is,

$$
\frac{\partial n_{e 1}}{\partial t}+n_{0}\left(\nabla \cdot v_{e 1}\right)+\left(v_{0} \cdot \nabla\right) n_{e 1}=0
$$

Linearising this we get

$$
n_{e 1}=\frac{k n_{0}}{\omega-k v_{0}} v_{e 1}=\left(-\frac{i e k n_{0}}{m\left(\omega-k v_{0}\right)^{2}} E+\frac{k^{2} \gamma_{e} K T_{e}}{m\left(\omega-k v_{0}\right)^{2}} n_{e 1}\right)
$$

Simplifying Equation (7) and Equation (9) we get,

and

$$
n_{i 1}=\frac{i e n_{0} k E}{M \omega^{2}-k^{2} \gamma_{i} K T_{i}}
$$

$$
n_{e 1}=\frac{-i e n_{0} k E}{m\left(\omega-k v_{0}\right)^{2}-k^{2} \gamma_{e} K T_{e}}
$$

The plasma waves leading to TSI are high frequency plasma oscillations. To deal with these type of waves it is well known that we should use Poisson's equations

$$
\epsilon_{0} \nabla \cdot \boldsymbol{E}=\rho
$$

i.e.,

$$
\epsilon_{0} \nabla \cdot \boldsymbol{E}_{1}=e\left(n_{i 1}-n_{e 1}\right)
$$

Here the electric field induced is due to the perturbation in the density. Assuming the perturbation as $\mathrm{e}^{i(k x-\omega t)} \hat{x}$ and substituting the value of $n_{i 1}$ and $n_{e 1}$ in Equation (13) and simplifying we obtain the following dispersion relation

$$
1=\omega_{p}^{2}\left[\frac{m / M}{\omega^{2}-k^{2} \frac{\gamma_{i} K T_{i}}{M}}+\frac{1}{\left(\omega-k v_{0}\right)^{2}-k^{2} \frac{\gamma_{e} K T_{e}}{m}}\right]
$$




\section{Results and Discussions}

Equation (14) is a fourth-order equation in $\omega$. and there will be four roots for this equation and each root represents a possible oscillation $\boldsymbol{E}_{1}=E \mathrm{e}^{i\left(k x-\omega_{j} t\right)} \hat{x}$, if all roots are real or if some roots are complex, they occur in complex conjugate pairs, and real part represents the propagation modes and imaginary part represents the instability. This could be analyzed by putting $\omega_{i}=\alpha_{i}+i \gamma_{i}$. Here $\alpha$ and $\gamma$ are the real part and imaginary part of frequency respectively. Now the oscillation is re expressed as

$$
\boldsymbol{E}_{1}=E \mathrm{e}^{i\left(k x-\alpha_{i} t\right)} \mathrm{e}^{\gamma_{i} t} \hat{x}
$$

Positive $\operatorname{Im}(\omega)$ denotes a growing wave and negative $\operatorname{Im}(\omega)$ indicates a damping wave.

As a check for knowing whether there is any unstable modes in the plasma, we follow the procedure in [9] and re-write the equation 14 as

$$
1=\frac{m / M}{x^{2}-a^{2} y^{2}}+\frac{1}{(x-y)^{2}-b^{2} y^{2}}
$$

where $a^{2}=\frac{v_{i}^{2}}{v_{0}^{2}}, b^{2}=\frac{v_{e}^{2}}{v_{0}^{2}}, x=\frac{\omega}{\omega_{p}}$ and $y=\frac{k v_{0}}{\omega_{p}} \cdot v_{i}^{2}=\frac{\gamma_{i} K T_{i}}{M}$ and $v_{e}^{2}=\frac{\gamma_{e} K T_{e}}{m}$ are the thermal velocities of ion and electron respectively. The dispersion relation shown in Equation (15) is a function of four variables $F(x, y, a, b)$. To make more clarity, it is better to discuss the cold plasma first and then extend to warm plasma. The disperssion relation for cold plasma is obtained by putting $a=b$ in Equation (15) and hence the dispersion relation becomes

$$
1=\frac{m / M}{x^{2}}+\frac{1}{(x-y)^{2}}=F(x, y)
$$

For any given $y$, we can plot $F(x, y)$ as a function of $x$. This function will have singularities at certain particular values of $x$ which can be found graphically. The singularities of cold plasma case is at $x=0$ and $x=y$. We can plot $F(x, y)$ as a function of $x$. The intersection of the curve with the line $F(x, y)=1$, gives the values of $x$ satisfying the dispersion relation. Since it is a fourth power of equation, there must be four intersection for four roots if all roots are real. Anything less than four intersection signifies the instability. The first graph is plotted to the value of $y=4$ and second graph is for $y=1$ shown in Figure 1 .

We could see four intersection in the first graph, which means the plasma is stable. But for in the second graph, there is only two intersection means the plasma is unstable, for $y=1$. As we know that $y=\frac{k v_{0}}{\omega_{p}}$, i.e,

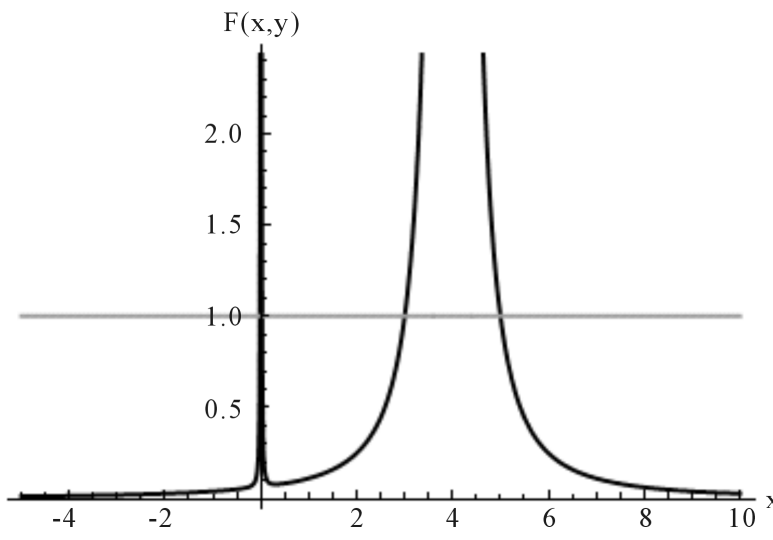

(a)

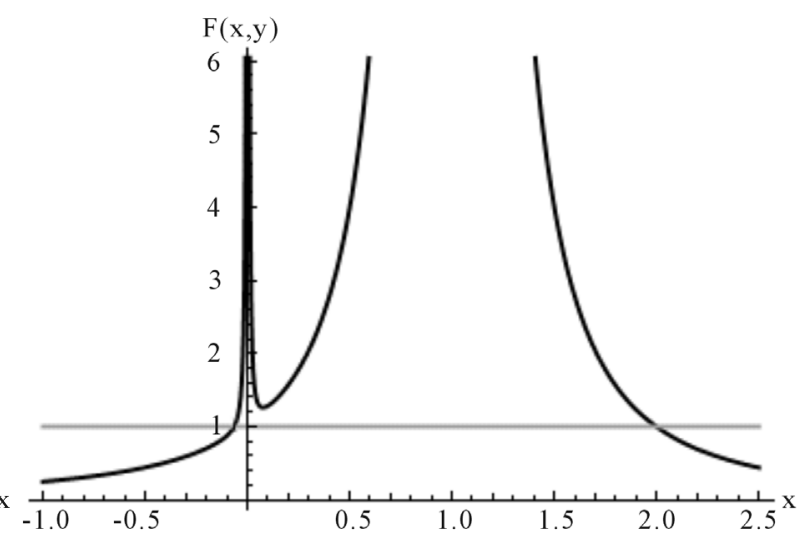

(b)

Figure 1. Figure shows the streaming instability for cold plasmas. The first graph (a) shows the intersection of $F(x, y)$ with the line $F(x, y)=1$, with four intersections and hence no imaginary roots for a higher value of $y=4$. The second graph shows the case of $y=1$ with two intersections and hence shows instability. 
when $k v_{0}$ is less than or equal to $\omega_{p}$ (the plasma frequency), the plasma becomes unstable. This could understood as that, when the frequency of induced oscillations is less than the plasma oscillation, the plasma derives the energy which could be probably at the expense of the energy of plasma waves. When the frequency is higher than the plasma frequency $(y>1)$ the plasma becomes stable because the the oscillation does not grow due to the lack of internal energy to support it.This shows that the cold plasma is stable for large values of $y$, but for sufficiently small values of drift velocity the plasma becomes unstable.

Now we can look into the dispersion relation of warm plasma, as it is to compare with the cold plasma, the plot is done for $y=4$ in which the cold plasma is stable for this value. Since we have additional two more parameters, we want to see the effect of a and b separately, so we neglect the thermal effect of electron by $\left(T_{e}=0\right)$ by setting $b=0$ and a is not equal to zero, shown in Figure 2.

We see the plasma is unstable with finite ion thermal velocity even without any thermal velocity of electron. For any higher values of $b$ the plot is same and hence the plasma shows instability. This is quite interesting that the ion temperature becomes a source of instability without the factor of considering the electron temperature. The additional mobility given to the ions by the temperature, negate the deficit of the density due to the high drift speed of electrons or the moving ions could easily find the sufficient electrons in the new spatial situation which sustains the field.

Also this could be verified by looking in to the Poissons equation that the electric field induced is depends on the perturbation density of ions and electrons. If we look into Equation (10)

$$
n_{i 1}=\frac{i e n_{0} k E}{M \omega^{2}-k^{2} \gamma_{i} K T_{i}}
$$

we see the second term in the denominator is with negative sign, which shows that the density of ions is increased by the temperature ions and hence the ion temperature becomes the source of instability. This could also viewed as the electric field induced by the drift of steaming electrons accelerate the ions to maintain the instability or the damping waves heats the ions.

Then we wish to analyze the effect of electron temperature, we set the ion temperature zero $(a=0)$ and plot for different values of $\mathrm{b}$, which is shown in Figure 3 .

Figures 3(a)-(c) show the plot for the value of $y=4$ and with zero ion temperature with different values of $b=1,2,3$. The first two graph shows that the plasma is stable for the ratio of the thermal velocity of electron to drift velocity till the value 2 , but when the thermal velocity becomes more than twice the value of drift, the plasma becomes unstable (shown in the third graph Figure 3(c)). This suggests that the minimum thermal velocity to ignite the instability must be greater than twice of the drift velocity. This result could be analyzed by little more detailed way by looking in to the result of cold plasma, we see from the figure the cold plasma is unstable for lower values of drift speed ( $y=\frac{k v_{0}}{\omega_{p}}, y=1$, shown in Figure 2). The plasma oscillations

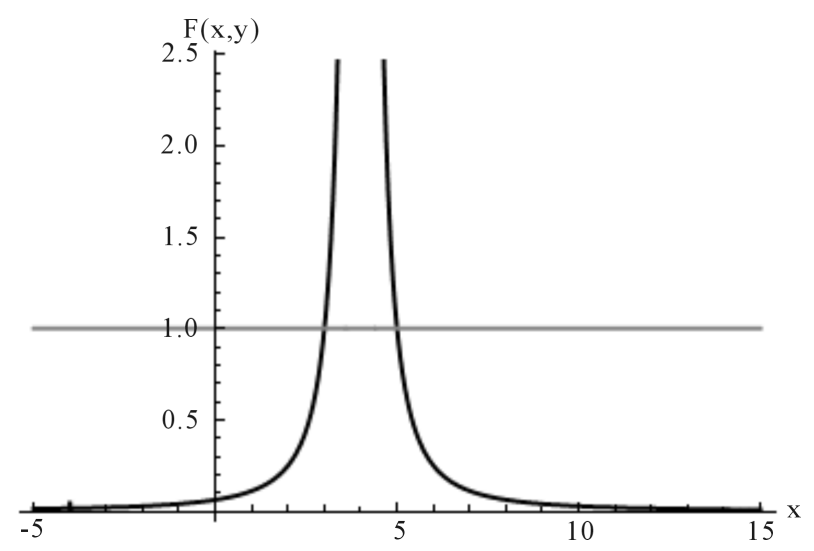

Figure 2. The streaming instability of hot plasmas is shown here.

The figure is for $y=4, a=1$ respectively and $b\left(\frac{v_{\text {the }}}{v_{0}}\right)=0$. 


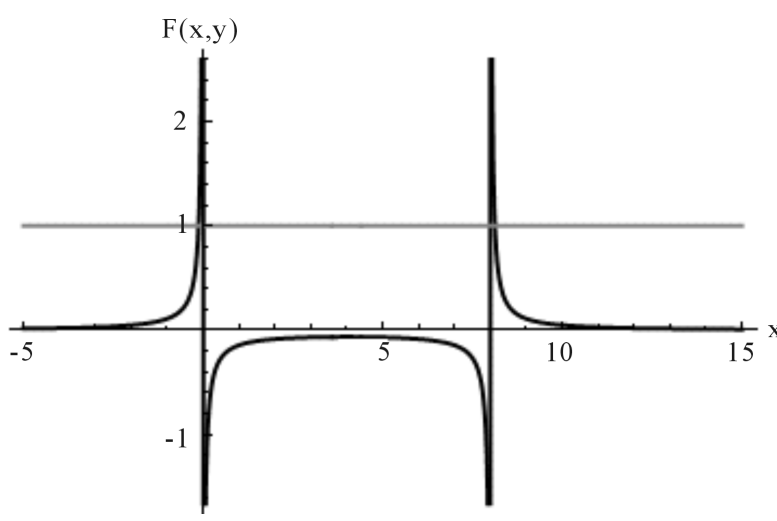

(a)

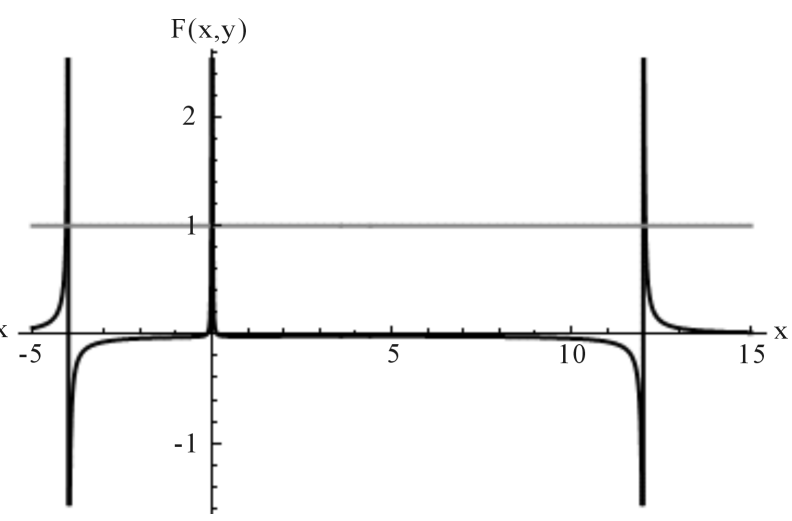

(b)

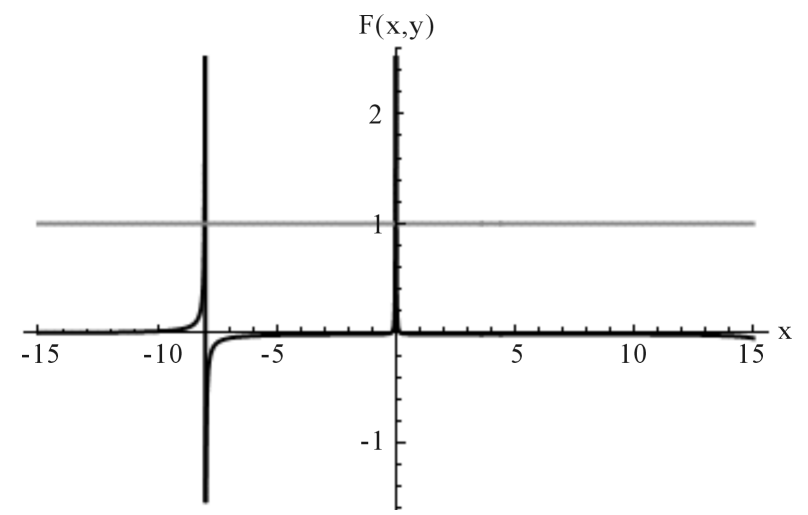

(c)

Figure 3. The streaming instability of hot plasmas is shown here. First three graphs (a), (b), and (c) are for $y=4$ at normalized electron thermal speeds $\frac{v_{\text {the }}}{v_{0}}=1,2,3$ respectively with the ion temperature zero.

depends on the sustainability of the electric field, which requires the charge neutrality conditions should be maintained for the oscillations. When the drift velocity of electrons increases, the plasma loses the charge neutrality conditions or local energy is reduced and hence the oscillations easily die out for larger drift speed. When the thermal electrons are added, it gives sufficient background density to support the electric field or it gives an additional local energy to maintain the oscillations. This could be verified from the equation, i.e., density of perturbation of electrons given as

$$
n_{e 1}=\frac{-i e n_{0} k E}{m\left(\omega-k v_{0}\right)^{2}-k^{2} \gamma_{e} K T_{e}}
$$

If we look into the equation, we see the second term in the denominator contains the square of drift speed which decreases the electron density, but the last term in the denominator is the kinetic pressure term it also reduces the density of electrons. So, it justifies the result that drift velocity of electrons and electron temperature reduces the instability.

As we have seen that the instability is sensitive to the drift speeds and thermal speeds, we have to analyze the growth rate for various temperatures. For deducing the growth rate, expand Equation 16, we arrive at the following fourth power equation. We know that for sufficiently small $y$, that is the plasma is unstable for $k v_{0} \approx y \omega_{p e}$ [5]. The dispersion relation can be written in the following form,

$$
1-\frac{\omega_{p e}^{2}(m / M)}{\left(\omega^{2}-\omega_{p e}^{2} a^{2} y^{2}\right)}-\frac{\omega_{p e}^{2}}{\left(\omega-y \omega_{p e}\right)^{2}-\omega_{p e}^{2} b^{2} y^{2}}=0
$$

i.e., 


$$
1-\frac{\omega_{p i}^{2}}{\left(\omega^{2}-\omega_{p e}^{2} a^{2} y^{2}\right)}-\frac{1}{\left(y-\frac{\omega}{\omega_{p e}}\right)^{2}-b^{2} y^{2}}=0
$$

Since $\frac{1}{\left(y-\frac{\omega}{\omega_{p e}}\right)^{2}-b^{2} y^{2}} \approx \frac{1}{y^{2}}\left(1+\frac{2 \omega}{y \omega_{p e}}\right)+\frac{1}{y^{2}} \frac{b^{2} y^{2}\left(1+\frac{2 \omega}{y \omega_{p e}}\right)}{-b^{2} y^{2}+\left(y^{2}-\frac{2 y \omega}{\omega_{p e}}\right)}$, the dispersion relation can be brought to the form,

$$
-\frac{\omega_{p i}^{2} y^{2}}{\omega^{2}-\omega_{p e}^{2} a^{2} y^{2}}+\left(y^{2}-1\right)-\frac{2 \omega}{y \omega_{p e}}-\frac{b^{2} y^{2}\left(1+\frac{2 \omega}{y \omega_{p e}}\right)}{-b^{2} y^{2}+\left(y^{2}-\frac{2 y \omega}{\omega_{p e}}\right)}=0
$$

After some simplifications and, the dispersion relation can be brought to the form

$$
\begin{aligned}
& 4 x^{4}-2 y^{3} x^{3}+\left[y^{4}-y^{2}\left(1+b^{2} y^{2}+4 a^{2}\right)\right] x^{2}+2 y^{3}\left[\frac{m}{M}+a^{2} y^{2}\right] x \\
& +y^{2}\left[y^{2}\left\{\frac{m}{M}\left(b^{2}-1\right)+a^{2}\left[1+\left(b^{2}-1\right) y^{2}\right]\right\}\right]=0,
\end{aligned}
$$

This is the general dispersion relation for warm plasma for two stream instability. This is a forth power equation in $x\left(\omega / \omega_{p}\right)$, has four roots and one of these roots shows high growth rate growth. The growth rate is plotted for coronal plasmas. Here also we want to check the influence of the electron and ion temperatures separately.

Figure 4(a) gives the growth rate for the different values of a by keeping $b$ as zero. As our earlier discussion on the influence of ion temperature on the instability, this graph clearly shows that the growth rate of the instability region shifted to the higher values of $y\left(k v_{0}\right)$, which is high frequency oscillations when ion temperature increases. As this is a high frequency oscillations which is driven by the ion temperature will be an effective way to heat the ions by the inducing these oscillations. This result could be more confirmed by analyzing the graph Figure 4(b) is a plot for different electron temperature with ion temperature zero. Here, in this graph, the growth rate shifts to the low frequency region for higher and higher electron temperature. Also it is to be noted that the growth rate is found only for high value of $b$ which is greater than 2 , as it is discussed earlier. The instability arises only when the kinetic energy is twice the drift energy as we know that for higher drift the plasma is stabilized, so any increase in the kinetic energy will be used for increasing the kinetic energy of ions to maintain the oscillations.

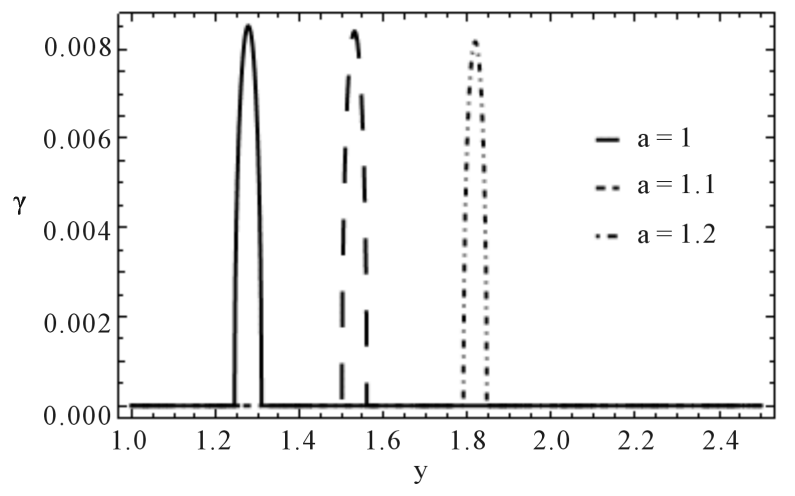

(a)

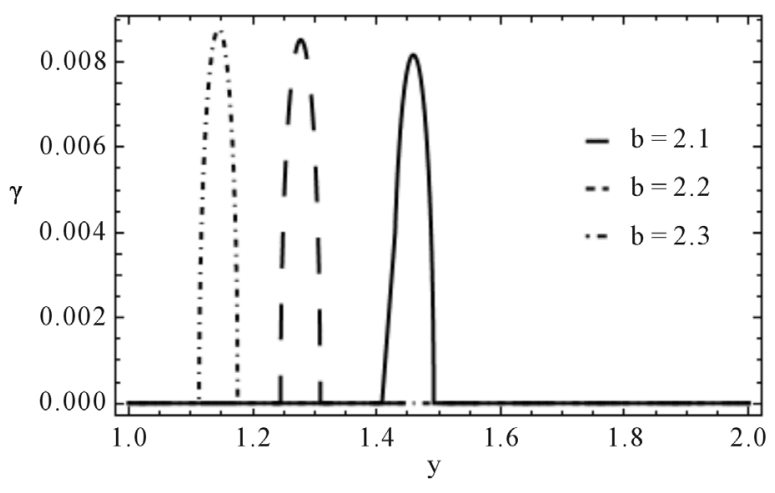

(b)

Figure 4. (a) for different $a$ values with $a=0$; (b) for different b values with $a=0$. 


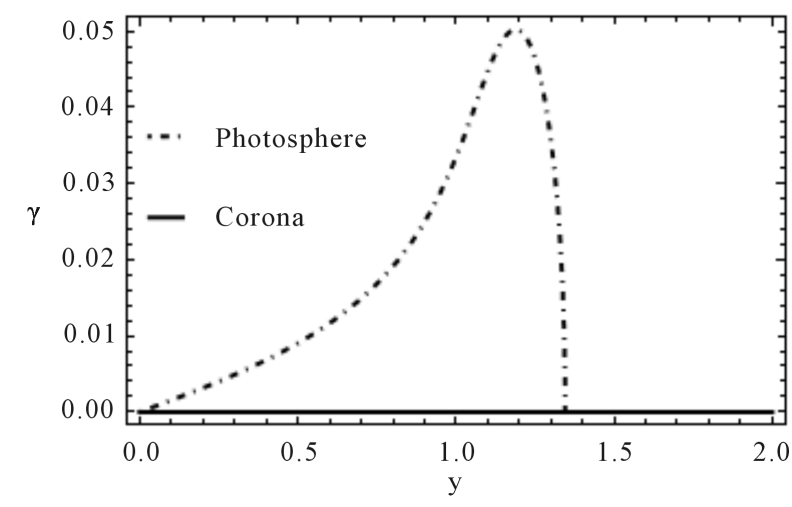

Figure 5. Plotted for drift velocity $v_{0}=10^{6}$.

To check the effect these waves over the solar atmosphere, we plot the growth rate for photosphere and corona and found the results are as expected shown in Figure 5. The high growth rate of photosphere plasma assures the possibility of such oscillations [1] [2] and these waves could drive the ions and these waves are damped out in the coronal plasma, which confirms the heating ions by these waves in the corona.

\section{Conclusions}

Streaming instabilities arise when there is relative velocity between ions and electrons in a plasma. The simplest type of streaming instability is the two stream instability which arises in an electron-proton plasma with electrons moving faster relative to ions. This instability has been studied by several authors for cold plasma, but for warm plasma, it is the first time that a study has been done by deriving a general dispersion relation. The dispersion relation for TSI is a fourth power equation in the angular frequency and wave vector, which has been studied for different conditions. For cold plasma, this instability arises only for lower values $k v_{0}$, which is less than the plasma frequency $\omega_{p}$, and hence the growth rate is very small, which is proportional to $(m / M)^{1 / 3}$ where $m$ is the mass of electron and $M$ is the mass of proton. But for warm plasma, it is unstable for higher frequencies greater than the plasma frequency, which shows a good growth rate.

This instability sensitively depends on the ion temperature, the growth rate shifts to the higher, and higher frequency region as the ion temperature increases. The high frequency oscillations are necessary conditions or pre-assumptions for this instability, that is the ion temperature become the source of this instability or the reverse can be more sensible that the damping of this instability will heat the ions.

Also, this instability depends on the electron temperature; the thermal velocity of electrons must be greater than a critical value which is double of the drift velocity, which means that the kinetic energy of electrons must be double times to negate the loss of the energy due to the drift velocity. It is found that the growth rate shifts towards the low frequency regions when the electron temperature increases. This is also quite exciting as it is a high frequency oscillation, if the kinetic energy of electrons shifts the growth rate to the low frequency region, to conserve the energy; the ions must be heated at the expense of the kinetic drift of electrons. This is quite true from the study when the drift velocity increases the instability decreases.

We have studied this instability for the coronal plasma and photospheric plasma, and found the result as predicted that there is no growth rate for coronal plasmas; the oscillations are damped out to heat the ions but for photosphere there is a good growth rate. This instability is a common one and hence this result can be applied to any hot electron-proton plasma and can be more useful to fusion devices.

\section{Acknowledgements}

The authors acknowledge the financial help made by the UGC for this work as a minor project on "Role of Macro Instabilities in Solar Coronal Heating".

\section{References}

[1] Aschwanden, M.J. (2005) Physics of Solar Corona. Springer, Berlin. 
[2] Thejappa, G., MacDowall, R.J., Bergamo, M. and Papadopoulos, K. (2012) Evidence for the Oscillating Two Stream Instability and Spatial Collapse of Langmuir Waves in a Solar Type III Radio Burst. Astrophysical Journal, 747, L1. http://dx.doi.org/10.1088/2[041-8205/747/1/L1

[3] Winglee, R.M. (1989) Heating and Acceleration of Heavy Ions during Solar Flares. Astrophysical Journal, 343, 511525. http://dx.doi.org/10.1086/167726

[4] Brown, J.C., Karlicky, M., MacKinnon, A.L., et al. (1990) Beam Heating in Solar Flares-Electrons or Protons? Astrophysical Journal, 73, 343-348. http://dx.doi.org/10.1086/191470

[5] Dwivedi, B.N. and Narain, U. (2006) Physics of Sun and Its Atmosphere. Proceedings of the National Workshop (India) on Recent Advances in Solar Physics, Meerut, November 2008.

[6] Sturrock, P.A., Holzer, T.E., Mihalas, D.M. and Ulrich, R.K. (1986) Physics of the Sun. Vol. 2, D. Reidel Publishing Company, Dordrecht.

[7] Tautz, R.C. and Schlickeiser, R. (2005) Covariant Kinetic Dispersion Theory of Linear Waves in Anisotropic Plasmas. III. Counterstreaming Plasmas. Physics of Plasmas, 12, Article ID: 072101. http://dx.doi.org/10.1063/1.1939967

[8] Tautz, R.C. and Schlickeiser, R. (2005) Counterstreaming Magnetized Plasmas. I. Parallel Wave Propagation. Physics of Plasmas, 12, Article ID: 122901. http://dx.doi.org/10.1063/1.2139505

[9] Tautz, R.C., Schlickeiser, R., (2006) Counterstreaming Magnetized Plasmas. II. Perpendicular Wave Propagation. Physics of Plasmas, 13, Article ID: 062901. http://dx.doi.org/10.1063/1.2207588

[10] Chen, F.F. (1981) Introduction to Plasma Physics and Controlled Fusion. Plenum Press, New York.

[11] Nicholson, D.R. (1983) Introduction to Plasma Theory. Wiley, Hoboken.

[12] Treumann, R.A. and Baumjohann, W. ( 2001) Advanced Space Plasma Physics. Imperial College Press, London. 